\title{
Computer-assisted initial diagnosis of rare diseases
}

\author{
Rui Alves ${ }^{\text {Corresp., }}$ 1,2 , Marc Piñol ${ }^{3}$, Jordi Vilaplana ${ }^{3,4}$ ， Ivan Teixidó ${ }^{3,4}$, Joaquim Cruz ${ }^{1,2}$, Jorge Comas ${ }^{1,2,3,4}$, \\ Ester Vilaprinyo ${ }^{1,2}$, Albert Sorribas ${ }^{1,2}$, Francesc Solsona ${ }^{3,4}$ \\ ${ }^{1}$ Departament de Cienciès Mèdiques Bàsiques, Universitat de Lleida, Lleida, Catalunya, Spain \\ 2 IRBLleida, Lleida, Catalunya, Spain \\ 3 Departament d'Informàtica i Enginyeria Industrial, Universitat de Lleida, Lleida, Catalunya, Spain \\ 4 INSPIRES, Lleida, Catalunya, Spain \\ Corresponding Author: Rui Alves \\ Email address: ralves@cmb.udl.es
}

Introduction: Most documented rare diseases have genetic origin. Because of their low individual frequency, an initial diagnosis based on phenotypic symptoms is not always easy, as practitioners might never have been exposed to patients suffering from the relevant disease. It is thus important to develop tools that facilitate symptom-based initial diagnosis of rare diseases by clinicians. In this work we aimed at developing a computational approach to aid in that initial diagnosis. We also aimed at implementing this approach in a user friendly web prototype. We call this tool Rare Disease Discovery. Finally, we also aimed at testing the performance of the prototype. Methods: Rare Disease Discovery uses the publicly available ORPHANET data set of association between rare diseases and their symptoms to automatically predict the most likely rare diseases based on a patient's symptoms. We apply the method to retrospectively diagnose a cohort of 187 rare disease patients with confirmed diagnosis. Subsequently we test the precision, sensitivity, and global performance of the system under different scenarios by running large scale Monte Carlo simulations. All settings account for situations where absent and/or unrelated symptoms are considered in the diagnosis. Results: We find that this expert system has high diagnostic precision ( $\geq 80 \%$ ) and sensitivity ( $\geq 99 \%$ ), and is robust to both absent and unrelated symptoms. Discussion: The Rare Disease Discovery prediction engine appears to provide a fast and robust method for initial assisted differential diagnosis of rare diseases. We coupled this engine with a user-friendly web interface and it can be freely accessed at http://disease-discovery.udl.cat/ . The code and most current database for the whole project can be downloaded from https://github.com/Wrrzag/DiseaseDiscovery/tree/no_classifiers. 
1

2

3

4

5

6

7

8

9

10

11

12

13

14

15

16

17

18

19

20

21

\section{Computer-Assisted Initial Diagnosis of Rare Diseases}

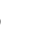

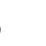

$$
\begin{aligned}
& \text { Rui Alves }{ }^{1,2, *, \S} \text {, Marc Piñol, }{ }^{3, *} \text {, Jordi Vilaplana }{ }^{3,4} \text {, Ivan Teixido }{ }^{3,4} \text {, Joaquim Cruz }{ }^{1,2} \text {, Jorge Comas }{ }^{1,2,3,4} \text {, } \\
& \text { Ester Vilaprinyo }{ }^{1,2} \text {, Albert Sorribas }{ }^{1,2} \text {, Francesc Solsona }{ }^{3,4, \S} \text {, }
\end{aligned}
$$

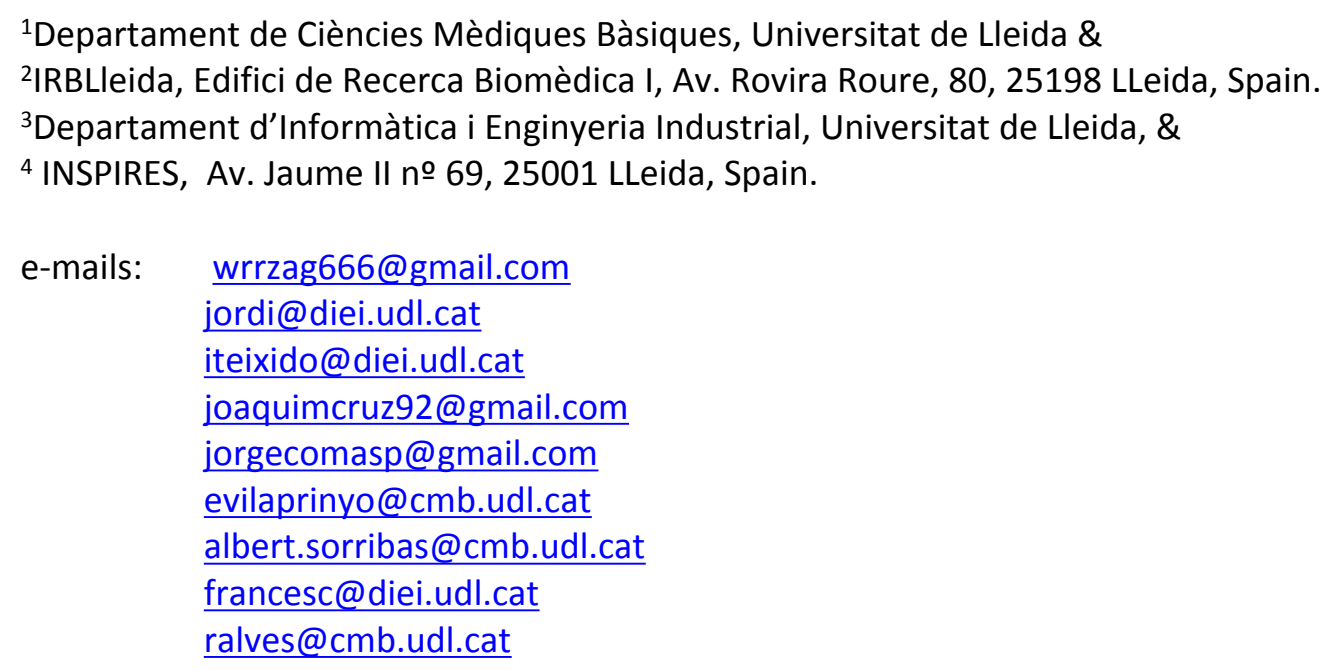

Francesc Solsona: Departament d'Informàtica i Enginyeria Industrial, Universitat de Lleida, Av. Jaume II no 69,

25001 LLeida, Spain.

email: francesc@diei.udl.cat

tel: 0034973702735

fax: 0034973702702

Rui Alves: Departament de Ciències Mèdiques Bàsiques \& IRBLleida, Universitat de Lleida, Edifici de Recerca Biomèdica I, Av. Rovira Roure 80, 25198 LLeida, Spain.

email: ralves@cmb.udl.cat

tel: 0034973702425

fax: 0034973702426 


\section{ABSTRACT}

40 Introduction: Most documented rare diseases have genetic origin. Because of their low individual

41 frequency, an initial diagnosis based on phenotypic symptoms is not always easy, as practitioners might never have been exposed to patients suffering from the relevant disease. It is thus important to develop tools that facilitate symptom-based initial diagnosis of rare diseases by clinicians. In this work we aimed at developing a computational approach to aid in that initial diagnosis. We also aimed at implementing this approach in a user friendly web prototype. We call this tool Rare Disease Discovery. Finally, we also aimed at testing the performance of the prototype.

Methods: Rare Disease Discovery uses the publicly available ORPHANET data set of association between rare diseases and their symptoms to automatically predict the most likely rare diseases based on a patient's symptoms. We apply the method to retrospectively diagnose a cohort of 187 rare disease patients with confirmed diagnosis. Subsequently we test the precision, sensitivity, and global performance of the system under different scenarios by running large scale Monte Carlo simulations. All settings account for situations where absent and/or unrelated symptoms are considered in the diagnosis.

Results: We find that this expert system has high diagnostic precision ( $\geq 80 \%)$ and sensitivity ( $\geq 99 \%)$, and

55 is robust to both absent and unrelated symptoms.

Discussion: The Rare Disease Discovery prediction engine appears to provide a fast and robust method

57 for initial assisted differential diagnosis of rare diseases. We coupled this engine with a user-friendly web interface and it can be freely accessed at http://disease-discovery.udl.cat/. The code and most current database for the whole project can be downloaded from https://github.com/Wrrzag/DiseaseDiscovery/tree/no_classifiers. 
61

62

63 Keywords: Computer Assisted Diagnosis; Rare Diseases; eHealth; Family Doctors; User-friendly 64 webserver 


\section{INTRODUCTION}

A Rare or orphan disease affects a small fraction of the population. This fraction is less than 200000 individuals in the total population in the USA, less than 50000 individuals in Japan, and less than 2000 in Australia. In Europe diseases are rare if they affects less than 1 in every 2000 individuals [1-3]. Overall, more than 10000 such diseases have been documented [4-6], and about $10 \%$ of the population suffers from some rare disease [3]. Most known rare diseases have genetic origin [4, 5]. The association between specific diseases and the genes that might cause them can be found at the OMIM database [5].

Because of their low individual frequency, initial diagnosis of rare diseases by clinicians is not always easy [7]. Often, those clinicians might never have been exposed to patients suffering from the disease. In addition, as it can be seen in ORPHANET [8], many different diseases have a partially overlapping spectrum of symptoms that can confuse the diagnosis. In general, conclusive diagnosis for most rare diseases comes from a genetic test that identifies the genetic variations associated to that disease. These tests tend to be expensive and/or target a specific (small set of) disease(s). Given all these constraints, it is important to develop methods and tools to facilitate a quick and accurate symptombased initial diagnosis of rare diseases.

Symptom-based diagnosis is a pattern recognition/classification problem, where an accurate prediction (the correct disease) must be made, based on a set of input characteristics (the symptoms). This is a classical computational problem, and computer- assisted medical diagnosis (CAD) can have many forms [9]. CAD is routinely used in clinical image analysis [see for example [10]], although other applications, such as telemedicine, are also becoming frequent [11-13].

Symptom-based Differential Diagnosis (DDX) generators that assist medical doctors in automatically generating initial diagnosis have been originally developed in the mid nineteen eighties [14]. A recent comparative analysis of these methods reveals that their accuracy and sensitivity did not significantly 
88 improve since $[15,16]$. Nevertheless, DDX generators could in principle be an optimal solution for an

89 initial diagnosis of rare diseases. However, given that the cost-effectiveness of eHealth solutions appears

90 to be debatable [17-29], it seems important that using such a computer-based solution should either be

91 free or have a low cost.

92 Some DDX generators dedicated to assisting in the diagnosis of genetic diseases have been

93 previously developed. They permit associating symptoms to diseases that can also be rare. Examples

94 are Phenomizer [30, 31], FindZebra [32, 33], PhenoTips [34] , or Phenotip [35]. While the first two are

95 aimed at assisting in diagnosing any genetic disease from a list of symptoms provided by the user, the

96 latter two have a somewhat different purpose. PhenoTips mostly provides a framework to share and

97 analyze patient data between professionals. Once data is introduced, it can also be used for assisted

98 diagnosis. In contrast, Phenotip focuses on prenatal diagnosis, which limits the symptoms it uses to

99 those that can be obtained from pre-natal analysis methods. These four tools are freely available to the

100 medical community. Other free computational tools that assist in rare disease patient treatment and

101 management do exist. For example, RAMEDIS [36] provides a highly accurate and manually curated

102 resource of human variations and corresponding phenotypes for rare metabolic diseases. DiseaseCard

103 [37] provides a similar service, with automated curation. The office of Rare Diseases from the NIH [38,

$10439]$ is another useful resource for diagnosis and follow up of rare diseases. However, these tools do not

105 provide a DDX generator that allow for doctors to get quick differential diagnostic options of rare

106 diseases.

Developing a specific and freely accessible DDX generator for rare diseases requires two types of data. First, appropriate data sources associating specific symptoms to genetic diseases should be available. A highly curated, often updated, dataset containing information about the association between symptoms and rare diseases is available to the community at ORPHANET [8]. Second, a large 
111 and freely available golden standard dataset of rare disease patients is needed to test and validate the

112 DDX generator. Although many initiatives are collecting data for tens to hundreds of thousands of rare

113 disease patients (e.g. $[41,42])$, these dataset have yet to made publicly available.

114 With these constraints in mind we set out to develop a prototype rare disease DDX generator, which

115 we call Rare Disease Discovery. Using the ORPHANET dataset as a source of information regarding the 116 association between symptoms and rare diseases, we developed Rare Disease Discovery (RDD,

117 http://disease-discovery.udl.cat/). This free DDX generator prototype is specific for rare diseases and

118 automatically predicts the most likely rare diseases based on the known set of symptoms provided by 119 the user.

\section{METHODS}

\section{Data Sources \& Software}

123 A highly curated list of rare diseases, associated to their respective symptoms, was downloaded from ORPHANET [6] on September 2015. A MySQL database where each disease is associated to its symptoms was built. The web technology underlying RDD is described in detail in the Supporting Methods section of the Supporting Appendix. All calculations and experiments were done using local Mathematica 127 scripts.

\section{Diagnostic Score Function and Disease Ranking}

129 The goal of RDD is to estimate which are the most likely rare diseases a patient might suffer from, based on the symptoms shown by that patient and on the symptoms that are associated to each rare disease

131 in the ORPHANET dataset. To rank diseases and provide a differential diagnosis, RDD uses the scoring 132 function $D S_{i}$ from Eq 1. 
$133 D S_{i}=1-\frac{n}{\operatorname{Max}\left[S_{\text {User }} S_{\text {Disease } i}\right]}$

Eq. 1

134 In Eq 1, $S_{\text {user }}$ represents the number of symptoms provided by the user, $S_{\text {Disease } i}$ represents the number of

135 symptoms of disease $i$ stored in the database, and Max $\left[S_{\text {user }}, S_{\text {Disease }}\right]$ represents the largest number

136 between $S_{\text {user }}$ and $S_{\text {Disease i. }} n$ represents the number of symptoms that are different between the set

137 submitted by the user and the set associated to any given rare disease in the database. The fraction

$138 \frac{n}{\operatorname{Max}\left[S_{\text {User }}, S_{\text {Disease } i}\right]}$ is always smaller than two and larger than or equal to zero. If all symptoms submitted by

139 the user are the same as those from a disease and that disease has exactly the same symptoms as those

140 provided by the user, $n=0$. If the symptoms provided by the user and the symptoms associated to a

141 disease are all different and $S_{\text {User }}=S_{\text {Disease } i}, n=2$. Thus, $-1 \leq D S_{i} \leq 1$.

142 RDD differentially diagnoses a patient by letting the user choose the list of symptoms that are

143 relevant for the specific case of interest. Once this list is selected, RDD calculates $D S_{i}$ for all diseases

144 stored in the database. Then, RDD ranks the diseases in order of increasing $D S_{i}$, presenting the disease

145 with the highest score as the most likely. In the Supporting Methods section of the Supporting Appendix

146 we discuss the performance of other scoring functions and prediction methods that were tested and

147 discarded.

148

\section{Retrospective study of previously diagnosed rare disease patients}

150 We selected all usable patients with a confirmed rare disease diagnosis from the RAMEDIS [36]

151 collection of patients in order to retrospectively use their symptoms and evaluate the diagnostic

152 performance of RDD on a real set of patients. See Supporting Methods and Supporting Figure 1 in

153 Supporting Appendix for selection details.

154 Calculating Sensitivity and Accuracy of the DDX Predictions and Significance of 155 the $\mathrm{DS}_{\mathrm{i}}$ Score 
156 Monte Carlo simulations were used to calculate the precision $p$ and sensitivity $s$ of RDD. $p$ is given by Eq.

1572 and $s$ is given by Eq. 3:

$158 p=\frac{\text { number of correctly predicted rare diseases }}{\text { number of correctly predicted rare diseases }+ \text { number of incorrectly predicted rare diseases }}$

$159 \quad$ Eq. 2

$160 S=\frac{\text { number of correctly predicted rare diseases }}{\text { total number of rare diseases }}$

161

Eq. 3

162 A prediction is considered to be correct if the disease that is ranked by $D S_{i}$ as the most likely is the

correct disease.

164 The global performance of RDD was also calculated using the F1-Score, which is the harmonic mean of $p$ 165 and $s$ :

$166 \quad F 1-$ Score $=2 \frac{p \times s}{p+s}$

Eq. 4

In addition, Monte Carlo simulations were also used to calculate the statistical significance of the score $D S_{i}$. All simulations were done using Mathematica [40].

\section{Benchmarking the Rare Disease Discovery Algorithm}

171 RDD's performance was benchmarked using four sets of experiments, all run using Stochastic Monte-

172 Carlo simulations. These experiments are detailed in the Supporting Methods section of the Supporting

173 Appendix. The first experiment tested how a combination of unreported and unrelated symptoms

174 affects prediction outcome of the RDD algorithm. The second experiment tested how unreported

175 symptoms affect the prediction performance of the RDD algorithm. The third and fourth experiments

176 estimate the minimum value for $D S_{i}$ that can be considered to be statistically significant and the

177 minimum difference between $D S_{i}$ values that is statistically significant, respectively. 


\section{RESULTS}

\section{Using the Rare Disease Discovery Web}

181 RDD is available at http://disease-discovery.udl.cat/. A simple web interface is provided to the user

182 (Figure 1A). Users can search for individual symptoms by typing on the text field. Once the relevant 183 symptom is identified, it must be selected. The user can type and select as many symptoms as required.

184 Symptoms that are absent from the database are not accepted by the server. Once all relevant 185 symptoms are selected, pressing the "Submit Symptoms" will generate a ranked list of disease 186 predictions. The disease with the highest score is shown. If the score of the predicted disease is not 187 statistically significant, this is indicated by a symbol. Clicking the name of the disease will lead 188 the user to the ORPHANET webpage where s/he can look up more information about the disease. In 189 addition, when the user clicks the link "Predicted Disease", RDD unfolds the full list of ranked diseases in 190 the browser (Figure 1B). If the user clicks the name of any of the diseases, s/he will be taken to the RDD 191 webpage with the list of symptoms for that disease. On that page s/he can also find external links about 192 the disease at $\mathrm{NIH}$.

The alphabetically ordered list of rare diseases, together with its associated symptoms can be directly accessed by pressing the "Disease List" pane (Figure 1C). The alphabetically ordered list of symptoms, together with its associated diseases can be accessed by pressing the "Symptom List" pane

(Figure 1D). Users can also select symptoms directly from this pane and use them for prediction. NIH searches can be automatically launched for the disease or the symptom of interest.

\section{Illustrative examples of RDD usage}

199 To illustrate the use of RDD, we randomly selected 10 diseases from the database. For each disease, we:

1 - Calculate the number of symptoms associated to that disease in the database. 
2012 - Randomly select the order of those symptoms.

2023 - Use the first symptom in the list to predict the disease.

2034 - Calculate the $D S_{i}$ score and rank for the disease.

2045 - Use the first five symptoms in the list to predict the disease (see Supporting Table 1 in the

205 Supporting Appendix).

206 6-Calculate the $D S_{i}$ score and rank for the disease.

2077 - Identify the minimal number of symptoms that rank the original disease as the most likely

208 prediction, and the score associated to those symptoms.

209 Results are summarized in Table 1. We see that one symptom is not sufficient to correctly identify any of

210 the tested rare disease as the most likely. However, with 5 symptoms, 7 of the diseases are correctly

211 predicted (although with $D S_{i}$ scores below significance level), and 9 of the diseases are ranked among

212 the top-2 most likely.

\section{Retrospective Study of Previously Diagnosed Rare Disease Patients}

214 A small retrospective study was also performed to evaluate the performance of the RDD prototype. We

215 obtained short report cards for 187 anonymous patients from the RAMEDIS[36] database (see

216 Supporting Methods and Supporting Figure1 in Supporting Appendix for details on report cards and

217 patient selection). Symptoms were clearly itemized and described at best in $30 \%$ of the cards. In the

218 remaining cases descriptions could fit alternative symptoms. In such cases all alternative symptoms are

219 considered. Given that 111 of the patients have three or less reported symptoms, errors are expected to

220 be large. A clinician, with direct knowledge about the patient, is unlikely to introduce such errors in the

221 diagnostic process. In spite of the noise in the data set, RDD included the correct disease in the list of

222 predictions for 117 out of 187 patients. In 60\% of these patients, the clinically diagnosed disease was on

223 the top ten list of predictions (See text and Supporting Figure 2 in Supporting Appendix for details). This 
224 percentage goes up to $80 \%$ if we consider the top fifty predictions for each patient. We note that only

225 approximately $17 \%$ of the predictions had a score that was significant $(>0.5)$.

\section{Comparison to Other DDX Generators}

227 RDD's performance was also compared with that of other DDX engines that were freely available for

228 illustrative purposes. After searching through the literature and the programs analyzed in Bond et al.

229 [15], this limited us to our own RDD (RareDiseaseDiscovery), in addition to DiagnosisPro, ISABEL,

230 Phenomizer, and FindZebra. While DiagnosisPro and ISABEL are general DDX generator,DD, FindZebra

231 and Phenomizer are DDX generators that are specific for genetic diseases.

By using the same ten diseases with their associated symptoms described in columns two and

four of Supporting Table 1 of the Supporting Appendix, we asked each of the DDX generators to come

up with a diagnosis of the disease. Results are summarized in Table 2. ISABEL identifies the correct

disease as a possibility in three of the ten diseases. Diagnosis Pro identifies the correct disease as a

possibility in four of the ten diseases. FindZebra identifies correctly nine of the diseases. RDD and

Phenomizer identify the correct disease as a possibility in all ten cases.

was the possibility that the performance of RDD was inflated with respect to the other DDX generators.

240 To control for this we randomly selected ten patients from RAMEDIS for which RDD had included the correct diagnostic in the top ten list of predictions. Using the symptoms associated to each patient, we interrogated the five DDX engines using their default parameters and evaluated if the correct disease was diagnosed as a possibility. The performance of ISABEL and DiagnosisPro was significantly better in this experiment. These two DDX engines succeeded in identifying seven out of ten diseases. ISABEL performed slightly better than DiagnosisPro, as it identified three of the seven diseases among the first 
247 identified five of the nine correct diseases in its top ten of suggestions. FindZebra identified eight of the

248 nine correct diseases in its top ten of suggestions.

\section{Benchmarking the Rare Disease Discovery Prototype}

250 Four additional benchmark tests were needed to evaluate the effect of absent and unrelated symptoms

251 on the diagnostic performance of RDD under more realistic, well controlled conditions. The first

252 experiment measured the aggregate effect of absent and unrelated symptoms in predicting the correct

253 disease. The precision $p$, sensitivity $s$, and F1-Score of RDD were calculated (Figure 2). When no

254 symptoms are added or deleted $p, s$, and F1-Score are always 1 , and the correct disease is predicted

$255100 \%$ of the times. As the number of incorrect symptoms increases, $p$ decreases, while $s$ remains

256 approximately constant. Decreases in either $p$ or the F1-Score only becomes larger than $5 \%$ when the

257 number of symptoms that are randomly added or deleted is equal to or higher than 10 in most of the

258 patients.

259

260

261

262

263

264

The second experiment tested the effect of unreported/absent symptoms in predicting the correct disease. The precision $p$, sensitivity $s$, and F1-Score of RDD were calculated (Figure 3). When no symptoms are deleted $p, s$, and F1-Score are always 1 . The correct disease is predicted $100 \%$ of the times. As the number of patients with deleted symptoms increases, $p$ decreases, while $s$ remains approximately constant. Decreases in $p$ or F-Score only become larger than $5 \%$ for the sets where $75 \%$ of the symptoms are deleted in $50 \%$ or more of the patients.

The two final experiments were used to determine statistical significance of both, the value of the $D S_{i}$ score used to rank the diseases and the difference between two $D S_{i}$ scores. These experiments estimate that a score $D S_{i} \geq 0.5$ has a probability lower than 0.0001 of being obtained by choosing a random set of symptoms (see Supporting Methods and Supporting Figure 3 in Supporting Appendix). In addition, it also suggests that differences between $D S_{i}$ scores lower than 0.01 are significant ( $p$ - 
270 value $<0.001$ ), as long as more than three symptoms are simultaneously submitted to RDD. If only one

271 symptom is submitted, then two $D S_{i}$ scores must differ by more than 0.14 ( $p$-value<0.001). Further

272 details are given in Supporting Methods and Supporting Table2 of the Supporting Appendix.

$273 D S_{i}$ decreases sharply with noise in all performed experiments; however, even if $D S_{i}$ is below the

274 statistically significant level, it can still be used to accurately predict the correct rare disease, although

275 with a lower confidence (See Supporting Methods and Supporting Figure 4 in Supporting Appendix for

276 details).

\section{DISCUSSION}

\section{Rare Disease Discovery}

280

281

282

283

284

285

286

287

In the vast majority of cases, a definitive and precise diagnostic of a rare disease requires genetic tests. However, in order to direct patients towards the appropriate medical specialists, family doctors need to make a preliminary diagnosis of the potential rare diseases that are consistent with the symptoms observed in the patient. These symptoms result either from macroscopic phenotypic observations of the patient or on clinical parameters from generic biochemical tests. It is at this stage of the diagnosis that rare disease DDX generators are likely to be most useful. Here we present an approach to create such a DDX generator, Rare Disease Discovery. We implement that approach as a fast, free, and user-friendly web prototype for initial CAD of patients with suspected rare diseases. We also tested the performance of this prototype in the limited context of the datasets that are available to us.

RDD runs typically take a few seconds, depending on the number of symptoms selected by the user. In the limited conditions under which we could test it, RDD has high precision and sensitivity in our benchmark experiments, suggesting that its diagnostic performance might be robust to situations where not all symptoms have been identified or are directly related with the disease the user is trying to 
293 identify. Precision is less robust to these factors than sensitivity, because the number of false positives is

294 always orders or magnitude smaller than the number of true negatives. This makes precision decrease

295 with noise much more sharply than sensitivity. We also show that even when the ranking score $D S_{i}$ is

296 below significance level, the correct disease is frequently in the set of diseases with the top ten highest

$297 D S_{i}$. This is also observed in our retrospective studied of 187 previously diagnosed patients.

298 Nevertheless, we remark that testing the performance of the application on much larger, more diverse,

299 anonymized datasets of real patients is needed to validate RDD and its performance. To our knowledge,

300 such datasets are not freely available to the community at present, although they may exist (see below).

Finally, we note that the approach underlying RDD can in principle be extended to any set of

diseases. If one has a database associating symptoms to diseases, then one can test the same score

function we use and benchmark that score using tests that are similar to the ones performed for RDD, establishing limits of statistical significance for the score function in the context of that database.

\section{Comparing RDD to similar tools}

Interestingly, our illustrative examples of usage suggest that RDD, Phenomizer, and FindZebra have very

similar performances while accurately diagnosing rare diseases. RDD and Phenomizer have an equally database. FindZebra is almost as good, only missing a beta thalassemia diagnosis in one of the ten synthetic patients. This experiment evaluated if the disease used to generate the list of symptoms was

311 included in the list of possible diseases associated to those symptoms. When we randomly select real

312 patients from the RAMEDIS dataset and perform the same experiment, RDD performs slightly better

313 than the other two rare disease DDX tools. While RDD always proposes the disease that was clinically

314 diagnosed to the patient in its top ten of diagnosed diseases, FindZebra repeats this performance for

315 eight out of ten patients and Phenomizer for five out of ten patients. However, both FindZebra and 
316 Phenomizer provide the correct disease in its top 100 list of possibilities for nine out of the ten patients.

317 As expected, RDD, FindZebra, and Phenomizer significantly outperform DDX engines that where

318 designed for CAD of general diseases (ISABEL and DiagnosisPro). We note that these experiments were

319 run using the default settings of all programs. In the case of Phenomizer we also repeated the

320 experiments changing the similarity measure and the multiple testing procedure of the program.

321 However, the results remained qualitatively similar. To be more confident about the comparative

322 performance of the RDD prototype with respect to Phenomizer and FindZebra, a batch study for the one

323 hundred and eighty seven patients from the RAMEDIS dataset would have been desirable. However, a

324 level of access to these tools that would permit automating this study is not available to the general

325 public and this comparison could not be performed. RDD performs fairly well in this dataset, as the

326 clinically diagnosed disease was on the top ten (fifty) list of predictions for more than $60 \%$ (80\%) of the

327 patients.

We note the qualitatively different approach that these three tools take to ranking the list of possible diseases for a given set of symptoms. Phenomizer takes what we would call a purely statistical approach and calculates the probability that a subset of symptoms could be generated from the complete set of symptom of a disease simply by accident. FindZebra performs a similar analysis for the random occurrence of specific terms in web documents. In contrast, RDD ranks the diseases based on a normalized hamming-like distance between the list of symptoms provided by the user and the list of symptoms from every disease in its database. Internally, RDD establishes the likelihood that a given score is significant or not, informs the user about it, but does not use this significance in the ranking of diseases. We speculate that a meta-server combining RDD, Phenomizer, and FindZebra and providing a consensus diagnostic list would be more accurate than any of the three programs alone. To facilitate this possibility we provide the RDD code and databases as a GitHub project 339 [https://github.com/Wrrzag/DiseaseDiscovery/tree/no_classifiers]. 


\section{Limitations}

343 RDD could in the future be developed to become a quick way to assist in initial DDX of rare diseases. This

344 speed comes at the cost of constraining server functionality. For example, admissible symptoms are

345 restricted to those present in the database. This should not be a problem because the data regarding 346 association between rare diseases and symptoms we use comes from ORPHANET. It is well-organized

347 and extensively curated by medical experts. We observed that the improvement in the quality of the

348 ORPHANET annotated dataset leads to an improvement in the predictions made by RDD, as indicated by comparing the benchmark of the server with the ORPHANET data from 2014 to the benchmark of the server with the ORPHANET data from 2015 (see Supporting Appendix 1). However, these improvements are small, suggesting that the quality of the ORPHANET disease-symptom annotation is quite high and further improvements to that dataset might not have a significant influence in the performance of RDD.

It is clear that any rare disease that is not included in that database can not be identified by RDD. However, this is also true for all other computer-assisted DDX tools, such as Phenomizer, or FindZebra, which can only identify diseases that are in their respective databases. Overall, the architecture of RDD allows for an easy replacement of the ORPHANET dataset by any other more comprehensive or more adequate dataset that may become available in the future. remain until larger, more diverse datasets of patients are made freely available to the community. We 
362 a more thorough validation of this and other rare disease DDX prototypes. For example, CEMARA

363 reports having data for 235000 rare disease patients. However, an anonymized version of that data is

364 not readily available for public use. If, or when, such a database becomes available we will use it to

365 further validate and test RDD. In addition we are actively looking for clinical teams that are interested in

366 using the RDD prototype for testing.

367

\section{CONCLUSIONS}

369 Rare Disease Discovery is a high performance web prototype for CAD of rare diseases. Its diagnostic

370 performance appears to be robust to situations where not all symptoms have been identified or are

371 directly related with the disease the user is trying to identify. The diagnostic performance of the

372 prototype on a limited set of 187 rare disease patients was good. If this diagnostic performance could be

373 tested and confirmed on larger and more diverse sets of rare disease patients, RDD might potentially

374 become a helpful tool for initial assisted diagnosis of rare disease patients.

375 COMPETING INTERESTS

376 The authors declare no competing interests.

\section{ACKNOWLEDGEMENTS}

378 We thank Drs. Gerard Piñol, Javier Trujillano, and Montse Rue for a critical reading of the paper and

379 helpful suggestions. 
381 This work was partially supported by the MEyC under contracts TIN2011-28689-C02-02 and BFU2010-

38217704 and by Universitat de Lleida and Departament de Ciències Mèdiques Bàsiques with bridge grants

383 to RA. The authors are members of the research groups 2014-SGR163 and 2014-SGR243, funded by the

384 Generalitat de Catalunya. The funders had no role in study design, data collection and analysis, decision

385 to publish, or preparation of the manuscript.

386

387

388

389

390

391

392

393

394

395

396

397

398

399

400

401

402

403

404

405

406

407

408

409

410

411

412

413

414

\section{REFERENCES}

1. About Rare Diseases | www.eurordis.org [http://www.eurordis.org/about-rare-diseases]

2. Lavandeira A: Orphan drugs: legal aspects, current situation. Haemophilia 2002, 8:194-8.

3. Schieppati A, Henter J-I, Daina E, Aperia A: Why rare diseases are an important medical and social issue. Lancet 2008, 371:2039-41.

4. Rath A, Olry A, Dhombres F, Brandt MM, Urbero B, Ayme S: Representation of rare diseases in health information systems: the Orphanet approach to serve a wide range of end users. Hum Mutat 2012, 33:803-8.

5. McKusick V: Mendelian Inheritance in Man : A Catalog of Human Genes and Genetic Disorders - NLM Catalog - NCBI. 12th edition. Baltimore: Johns Hopkins University Press; 2008.

6. OrphaData [http://www.orphadata.org/cgi-bin/inc/product4.inc.php]

7. Polizzi A, Balsamo A, Bal MO, Taruscio D: Rare diseases research and practice. Endocr Dev 2014, 27:234-56.

8. Maiella S, Rath A, Angin C, Mousson F, Kremp O: [Orphanet and its consortium: where to find expert-validated information on rare diseases]. Rev Neurol (Paris) 2013, 169 Suppl :S3-8.

9. Eadie LH, Taylor P, Gibson AP: Recommendations for research design and reporting in computer-assisted diagnosis to facilitate meta-analysis. J Biomed Inform 2012, 45:390-7.

10. Wang S, Summers RM: Machine learning and radiology. Med Image Anal 2012, 16:933-51.

11. Lopman BA, Barnabas R V, Boerma JT, Chawira G, Gaitskell K, Harrop T, Mason P, Donnelly CA, Garnett GP, Nyamukapa C, Gregson S: Creating and validating an algorithm to measure AIDS mortality in the adult population using verbal autopsy. PLoS Med 2006, 3:e312.

12. Soyer HP, Hofmann-Wellenhof R, Massone C, Gabler G, Dong H, Ozdemir F, Argenziano G: telederm.org: freely available online consultations in dermatology. PLoS Med 2005, 2:e87. 
13. Steele AW, Eisert S, Witter J, Lyons P, Jones MA, Gabow P, Ortiz E: The effect of automated alerts on provider ordering behavior in an outpatient setting. PLoS Med 2005, 2:e255.

14. Barnett GO, Cimino JJ, Hupp JA, Hoffer EP: DXplain. An evolving diagnostic decision-support system. JAMA 1987, 258:67-74.

15. Bond WF, Schwartz LM, Weaver KR, Levick D, Giuliano M, Graber ML: Differential diagnosis generators: an evaluation of currently available computer programs. J Gen Intern Med 2012, 27:213-9.

16. Umscheid CA, Hanson CW: A follow-up report card on computer-assisted diagnosis-the grade: C+. J Gen Intern Med 2012, 27:142-4.

17. Black AD, Car J, Pagliari C, Anandan C, Cresswell K, Bokun T, McKinstry B, Procter R, Majeed A, Sheikh A: The impact of eHealth on the quality and safety of health care: a systematic overview. PLoS Med 2011, 8:e1000387.

18. Free C, Phillips G, Galli L, Watson L, Felix L, Edwards P, Patel V, Haines A: The effectiveness of mobile-health technology-based health behaviour change or disease management interventions for health care consumers: a systematic review. PLoS Med 2013, 10:e1001362.

19. Free C, Phillips G, Watson L, Galli L, Felix L, Edwards P, Patel V, Haines A: The effectiveness of mobile-health technologies to improve health care service delivery processes: a systematic review and meta-analysis. PLoS Med 2013, 10:e1001363.

20. McLean S, Sheikh A, Cresswell K, Nurmatov U, Mukherjee M, Hemmi A, Pagliari C: The impact of telehealthcare on the quality and safety of care: a systematic overview. $P L o S$ One 2013, 8:e71238.

21. Eysenbach G, Powell J, Kuss O, Sa E-R: Empirical studies assessing the quality of health information for consumers on the world wide web: a systematic review. $J A M A$, 287:2691-700.

22. Avery AJ, Rodgers S, Cantrill JA, Armstrong S, Cresswell K, Eden M, Elliott RA, Howard R, Kendrick D, Morris CJ, Prescott RJ, Swanwick G, Franklin M, Putman K, Boyd M, Sheikh A: A pharmacist-led information technology intervention for medication errors (PINCER): a multicentre, cluster randomised, controlled trial and cost-effectiveness analysis. Lancet 2012, 379:1310-9.

23. Free C, Knight R, Robertson S, Whittaker R, Edwards P, Zhou W, Rodgers A, Cairns J, Kenward MG, Roberts I: Smoking cessation support delivered via mobile phone text messaging (txt2stop): a single-blind, randomised trial. Lancet 2011, 378:49-55.

24. Howitt P, Darzi A, Yang G-Z, Ashrafian H, Atun R, Barlow J, Blakemore A, Bull AMJ, Car J, Conteh L, Cooke GS, Ford N, Gregson SAJ, Kerr K, King D, Kulendran M, Malkin RA, Majeed A, Matlin S, Merrifield R, Penfold HA, Reid SD, Smith PC, Stevens MM, Templeton MR, Vincent C, Wilson E: Technologies for global health. Lancet 2012, 380:507-35.

25. Sheikh A, Jha A, Cresswell K, Greaves F, Bates DW: Adoption of electronic health records in UK hospitals: lessons from the USA. Lancet 2014, 384:8-9.

26. Morrison C, Jones M, Jones R, Vuylsteke A: “You can”t just hit a button': an ethnographic study of strategies to repurpose data from advanced clinical information 
457

458

459

460

461

462

463

464

465

466

467

468

469

470

471

472

473

474

475

476

477

478

479

480

481

482

483

484

485

486

487

488

489

490

491

492

493

494

495

496

497

systems for clinical process improvement. $B M C \operatorname{Med} 2013,11: 103$.

27. Huckvale K, Car M, Morrison C, Car J: Apps for asthma self-management: a systematic assessment of content and tools. BMC Med 2012, 10:144.

28. Greenhalgh T, Swinglehurst D: Studying technology use as social practice: the untapped potential of ethnography. BMC Med 2011, 9:45.

29. Mazzucato M, Houyez F, Facchin P: The importance of helplines in National Plans. Orphanet J Rare Dis 2014, 9(Suppl 1):O12.

30. Köhler S, Doelken SC, Mungall CJ, Bauer S, Firth H V, Bailleul-Forestier I, Black GCM, Brown DL, Brudno M, Campbell J, FitzPatrick DR, Eppig JT, Jackson AP, Freson K, Girdea M, Helbig I, Hurst JA, Jähn J, Jackson LG, Kelly AM, Ledbetter DH, Mansour S, Martin CL, Moss C, Mumford A, Ouwehand WH, Park S-M, Riggs ER, Scott RH, Sisodiya S, Van Vooren S, Wapner RJ, Wilkie AO, Wright CF, Vulto-van Silfhout AT, de Leeuw N, de Vries BB, Washingthon NL, Smith CL, Westerfield M, Schofield P, Ruef BJ, Gkoutos GV, Haendel M, Smedley D, Lewis SE, Robinson PN.: The Human Phenotype Ontology project: linking molecular biology and disease through phenotype data. Nucleic Acids Res 2014, 42(Database issue):D966-74.

31. Köhler S, Schulz MH, Krawitz P, Bauer S, Dölken S, Ott CE, Mundlos C, Horn D, Mundlos $\mathrm{S}$, Robinson $\mathrm{PN}$ : Clinical diagnostics in human genetics with semantic similarity searches in ontologies. Am J Hum Genet 2009, 85:457-64.

32. Dragusin R, Petcu P, Lioma C, Larsen B, Jørgensen HL, Cox IJ, Hansen LK, Ingwersen P, Winther O: FindZebra: a search engine for rare diseases. Int J Med Inform 2013, 82:52838 .

33. Winther O, Svenstrup D, Henningsen PP, Kristiásson R, Jørgensen HL: FindZebra - the search engine for difficult medical cases. Orphanet $J$ Rare Dis 2014, 9(Suppl 1):O5.

34. Girdea M, Dumitriu S, Fiume M, Bowdin S, Boycott KM, Chénier S, Chitayat D, Faghfoury H, Meyn MS, Ray PN, So J, Stavropoulos DJ, Brudno M: PhenoTips: patient phenotyping software for clinical and research use. Hum Mutat 2013, 34:1057-65.

35. Porat S, de Rham M, Giamboni D, Van Mieghem T, Baud D: Phenotip - a web-based instrument to help diagnosing fetal syndromes antenatally. Orphanet J Rare Dis 2014, 9:204.

36. Töpel T, Scheible D, Trefz F, Hofestädt R: RAMEDIS: a comprehensive information system for variations and corresponding phenotypes of rare metabolic diseases. Hum Mutat 2010, 31:E1081-8.

37. Lopes P, Oliveira JL: An innovative portal for rare genetic diseases research: the semantic Diseasecard. $J$ Biomed Inform 2013, 46:1108-15.

38. Daneshvari S, Youssof S, Kroth PJ: The NIH Office of Rare Diseases Research patient registry Standard: a report from the University of New Mexico's Oculopharyngeal Muscular Dystrophy Patient Registry. AMIA Annu Symp Proc 2013, 2013:269-77.

39. Office of Rare Diseases Research (ORDR-NCATS) | Supports rare disease research and information [http://rarediseases.info.nih.gov/]

40. Wolfram S: The MATHEMATICA ${ }^{\circledR}$ Book, Version 4. Cambridge University Press; 1999.

41. Choquet R, Landais P: The French national registry for rare diseases: an integrated 
498 model from care to epidemiology and research. Orphanet J Rare Dis 2014, 9(Suppl 1):O7.

499 42. Koutouzov S: The European research area network - E-Rare. Orphanet J Rare Dis 500 2010, 5(Suppl 1):O15.

501

502

503 


\section{FIGURE CAPTIONS}

505 Figure 1 - The web interface for Rare Disease Discovery. A - Entry screen. Users can type and select

506 various symptoms. Once all relevant symptoms have been selected, the user can press "Submit

507 Symptoms". B - Example of a differential diagnosis provided by the program. C - List of diseases in the

508 database, with its associated symptoms. D - List of symptoms in the database, with its associated

509 diseases.

510 Figure 2 - Joint effect of unreported and unrelated symptoms on the predictive accuracy of Rare Disease

511 Discovery. A - Plot of F1-Score as a function of the \% of patients with a known rare disease where 1, 2,

$5123,4,5,10$, or 20 symptoms were randomly added or deleted. B - Plot of Precision as a function of the \%

513 of patients with a known rare disease where $1,2,3,4,5,10$, or 20 symptoms were randomly added or

514 deleted. C - Plot of Sensitivity as a function of the \% of patients with a known rare disease where 1, 2, 3,

$5154,5,10$, or 20 symptoms were randomly added or deleted. Without noise, the F1-Score is always 1 . The

516 F1-Score decreases as noise (\% of patients with deleted symptoms) increases. This is mainly due to a

517 decrease in precision. Sensitivity is always low because the number of false positives is always orders or

518 magnitude smaller than the number of true negatives. In the worst case scenario (20 incorrect

519 symptoms in $100 \%$ of the patients), the appropriate disease is contained in the set of diseases with the

520 highest score for more than $80 \%$ of the patients.

521 Figure 3 - Effect of unreported symptoms on the predictive accuracy of Rare Disease Discovery. A - Plot

522 of F1-Score as a function of the $\%$ of patients with a known rare disease where $25 \%, 50 \%$, and $75 \%$ of

523 the symptoms were randomly deleted. B - Plot of Precision as a function of the \% of patients with a

524 known rare disease where $25 \%, 50 \%$, and $75 \%$ of the symptoms were randomly deleted. C - Plot of

525 Sensitivity as a function of the $\%$ of patients with a known rare disease where $25 \%, 50 \%$, and $75 \%$ of the

526 symptoms were randomly deleted. Without noise (no deleted symptoms), the F1-Score is always 1 . The 
527 F1-Score decreases as noise (\% of patients with deleted symptoms) increases. This is mainly due to a

528 decrease in precision. Sensitivity is always low because the number of false positives is always orders or 529 magnitude smaller than the number of true negatives. In the worst case scenario $(75 \%$ deleted

530 symptoms in $100 \%$ of the patients), the appropriate disease is contained in the set of diseases with the 531 highest score for more than $90 \%$ of the patients.

\section{TABLE CAPTIONS}

Table 1 - Examples of prediction results for a randomly chosen set of ten rare diseases. Diseases are identified in column 1. Column 2 indicates the total number of symptoms associated to the disease in the ORPHANET dataset. Column 3 presents $D S_{i}$ for the disease when one symptom is submitted to RDD, as well as the ranking of the disease in the list of predictions. Column 4 shows $D S_{i}$ for the disease when five symptoms are simultaneously submitted to RDD, as well as the ranking of the disease in the list of predictions. Column 5 displays minimum $D S_{i}$ at which the disease is ranked as the most likely prediction, as well as the number of symptoms needed for that value of $D S_{i}$ to be obtained. Finally, column 6 indicates the number of symptoms that make $D S_{i} \geq 0.5$ for the disease, which is the value above which $D S_{i}$ is statistically significant. Details about the symptoms are given in Supporting Table 1 of the Supporting Appendix.

Table 2 - Comparison of predictions between DDX generators. Here we compare the most likely diagnosis of four well-known and freely available (at least for testing purposes) DDX generators with that provided by Rare Disease Discovery, when considering the joint symptoms used to perform the study summarized in columns 1 and 4 of Table 1.

Table 3 - Comparison of predictions between DDX generators. Here we compare the most likely diagnosis of four well-known and freely available (at least for testing purposes) DDX generators with 
549 that provided by Rare Disease Discovery. 10 patients with different symptoms and/or diseases were 550 randomly selected from the RAMEDIS dataset. All symptoms were used. 


\section{Additional Material:}

553

554 Supporting File 1 -Supporting_Appendix_1.docx: Appendix for "Efficient Computer-Assisted Diagnosis

555 of Rare Diseases Using Rare Disease Discovery" containing additional methods, results and figures. 


\section{1}

The web interface for Rare Disease Discovery.

A - Entry screen. Users can type and select various symptoms. Once all relevant symptoms have been selected, the user can press "Submit Symptoms". B - Example of a differential diagnosis provided by the program. C - List of diseases in the database, with its associated symptoms. D - List of symptoms in the database, with its associated diseases.

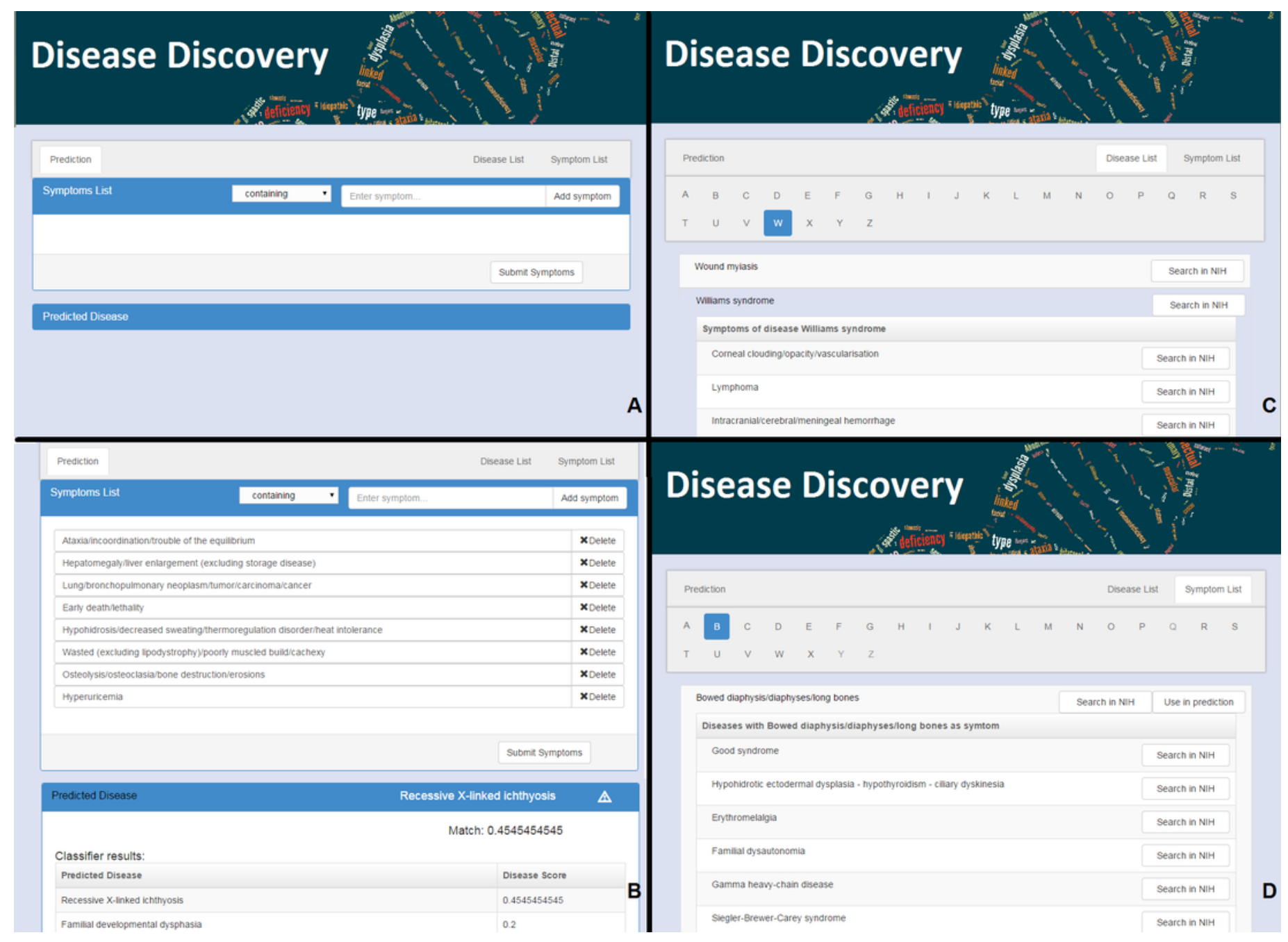




\section{2}

Joint effect of unreported and unrelated symptoms on the predictive accuracy of Rare Disease Discovery.

A - Plot of F1-Score as a function of the $\%$ of patients with a known rare disease where 1,2 , $3,4,5,10$, or 20 symptoms were randomly added or deleted. B - Plot of Precision as a function of the \% of patients with a known rare disease where $1,2,3,4,5,10$, or 20 symptoms were randomly added or deleted. C - Plot of Sensitivity as a function of the \% of patients with a known rare disease where $1,2,3,4,5,10$, or 20 symptoms were randomly added or deleted. Without noise, the F1-Score is always 1 . The F1-Score decreases as noise (\% of patients with deleted symptoms) increases. This is mainly due to a decrease in precision. Sensitivity is always low because the number of false positives is always orders or magnitude smaller than the number of true negatives. In the worst case scenario (20 incorrect symptoms in $100 \%$ of the patients), the appropriate disease is contained in the set of diseases with the highest score for more than $80 \%$ of the patients. 


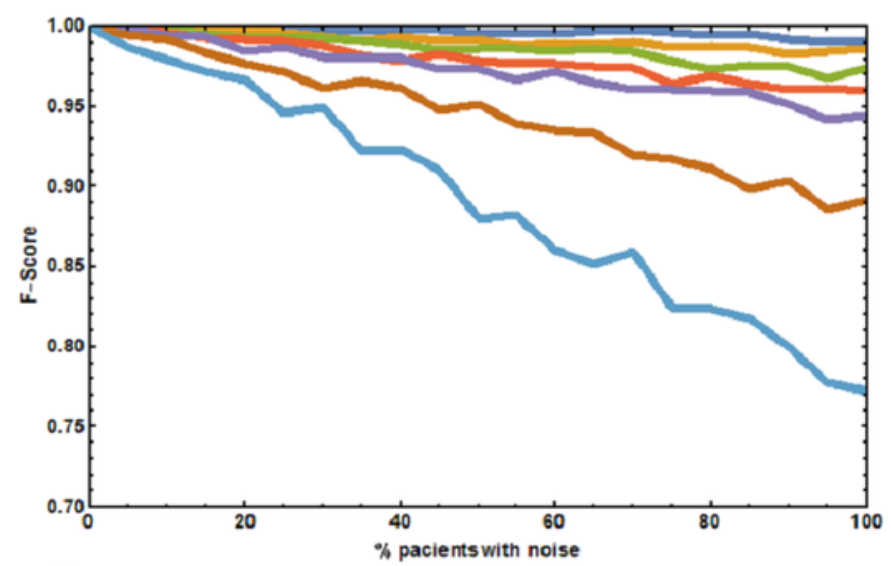

$$
\begin{aligned}
& -1 \text { symptom } \\
& -2 \text { symptom } \\
& -3 \text { symptom } \\
& -4 \text { symptom } \\
& -5 \text { symptom } \\
& -10 \text { symptom } \\
& -20 \text { symptom }
\end{aligned}
$$
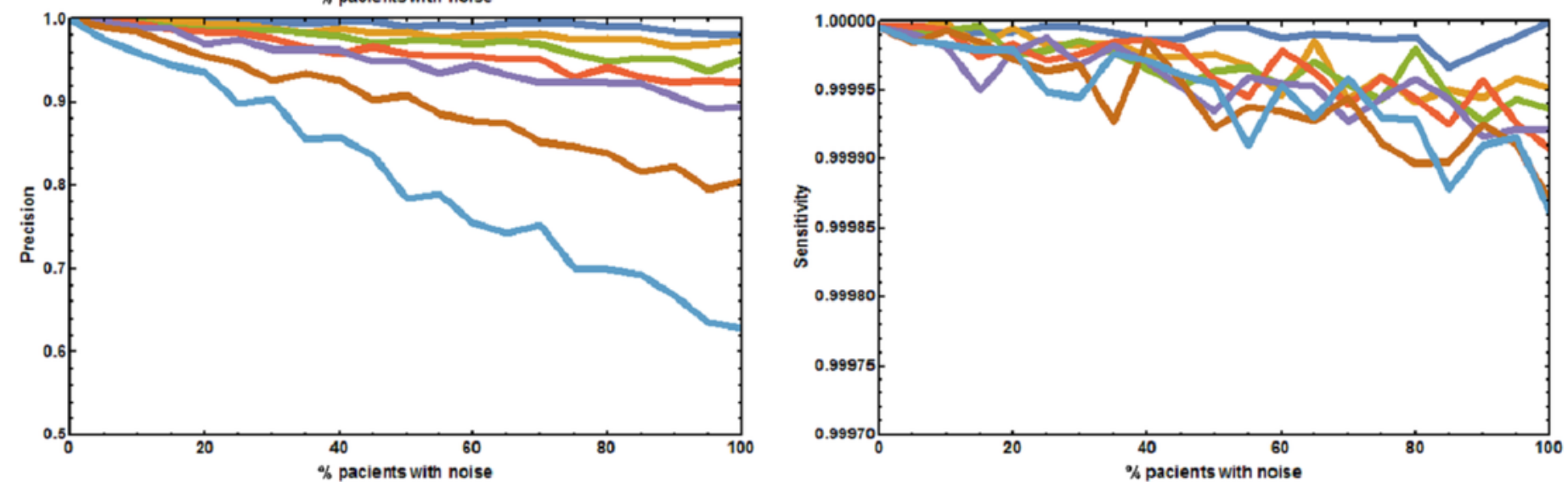


\section{3}

Effect of unreported symptoms on the predictive accuracy of Rare Disease Discovery.

A - Plot of F1-Score as a function of the $\%$ of patients with a known rare disease where $25 \%$, $50 \%$, and $75 \%$ of the symptoms were randomly deleted. B - Plot of Precision as a function of the $\%$ of patients with a known rare disease where $25 \%, 50 \%$, and $75 \%$ of the symptoms were randomly deleted. C - Plot of Sensitivity as a function of the $\%$ of patients with a known rare disease where $25 \%, 50 \%$, and $75 \%$ of the symptoms were randomly deleted. Without noise (no deleted symptoms), the F1-Score is always 1. The F1-Score decreases as noise (\% of patients with deleted symptoms) increases. This is mainly due to a decrease in precision. Sensitivity is always low because the number of false positives is always orders or magnitude smaller than the number of true negatives. In the worst case scenario (75\% deleted symptoms in $100 \%$ of the patients), the appropriate disease is contained in the set of diseases with the highest score for more than $90 \%$ of the patients.
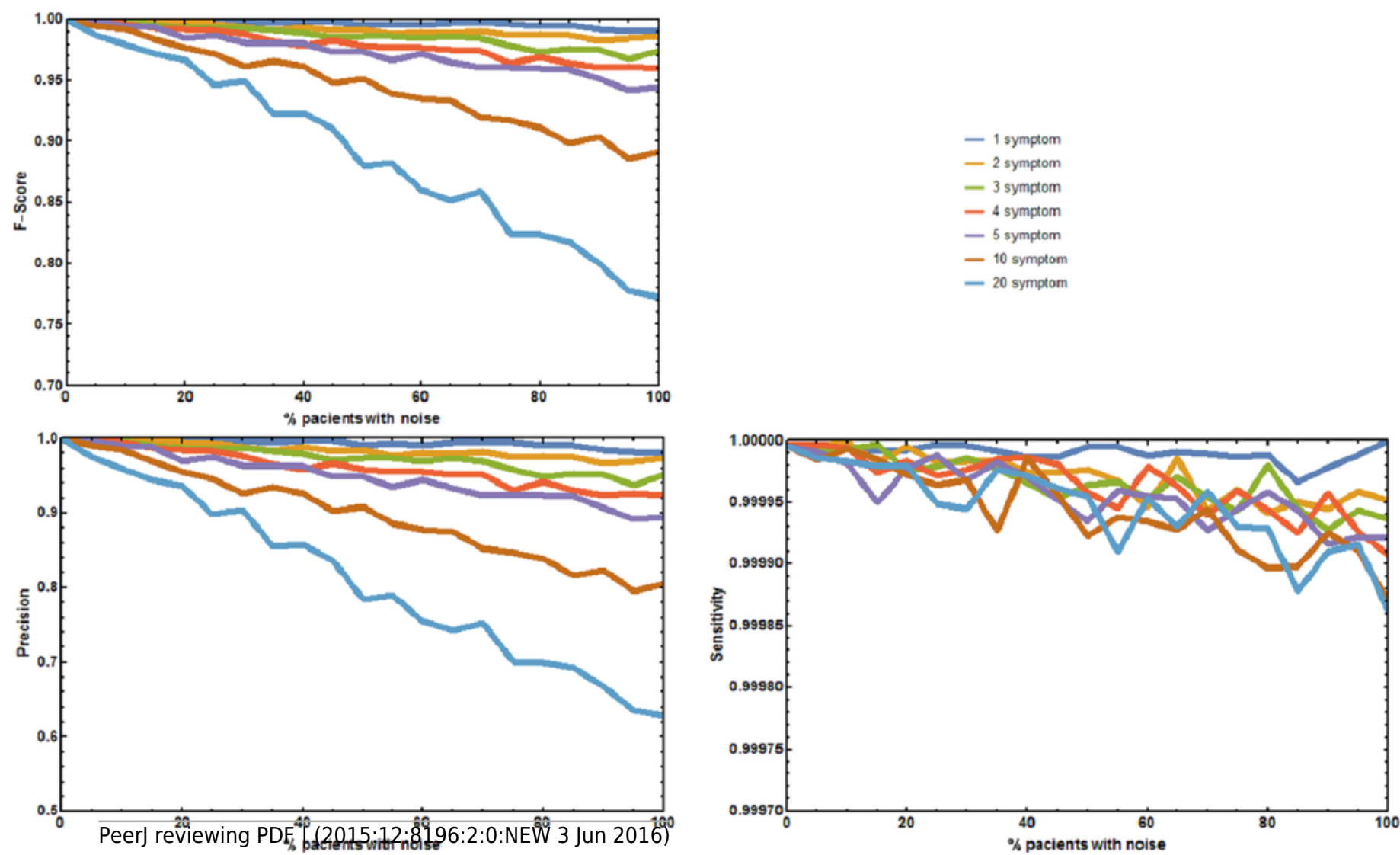


\section{Table $\mathbf{1}$ (on next page)}

Examples of prediction results for a randomly chosen set of ten rare diseases.

Diseases are identified in column 1. Column 2 indicates the total number of symptoms associated to the disease in the ORPHANET dataset. Column 3 presents $D S_{i}$ for the disease when one symptom is submitted to RDD, as well as the ranking of the disease in the list of predictions. Column 4 shows $D S_{i}$ for the disease when five symptoms are simultaneously submitted to RDD, as well as the ranking of the disease in the list of predictions. Column 5 displays minimum $D S_{i}$ at which the disease is ranked as the most likely prediction, as well as the number of symptoms needed for that value of $D S_{i}$ to be obtained. Finally, column 6 indicates the number of symptoms that make $D S_{i} \geq 0.5$ for the disease, which is the value above which is statistically significant. Details about the symptoms are given in Supporting Table 1 of the Supporting Appendix. 
1 Table 1 - Examples of prediction results for a randomly chosen set of ten rare diseases. Diseases are 2 identified in column 1 . Column 2 indicates the total number of symptoms associated to the disease in 3 the ORPHANET dataset. Column 3 presents $D S_{i}$ for the disease when one symptom is submitted to RDD, 4 as well as the ranking of the disease in the list of predictions. Column 4 shows $D S_{i}$ for the disease when 5 five symptoms are simultaneously submitted to RDD, as well as the ranking of the disease in the list of 6 predictions. Column 5 displays minimum $D S_{i}$ at which the disease is ranked as the most likely prediction, 7 as well as the number of symptoms needed for that value of $D S_{i}$ to be obtained. Finally, column 6 8 indicates the number of symptoms that make $D S_{i} \geq 0.5$ for the disease, which is the value above which $9 D S_{i}$ is statistically significant. Details about the symptoms are given in Supporting Table 1 of the 10 Supporting Appendix.

\begin{tabular}{|c|c|c|c|c|}
\hline Disease & $\begin{array}{l}\text { Number of } \\
\text { associated } \\
\text { symptoms }\end{array}$ & $\begin{array}{l}\text { Score at } 1 \\
\text { symptom } \\
\text { (rank) }\end{array}$ & $\begin{array}{l}\text { Minimun score } \\
\text { at rank } 1 \\
\text { (number of } \\
\text { symptoms) }\end{array}$ & $\begin{array}{l}\text { Number of } \\
\text { symptoms for } \\
\text { statistically } \\
\text { significant } \\
\text { score }\left(D S_{i}>0.5\right)\end{array}$ \\
\hline Beta-Thalassemia & 23 & $0.043\left(67^{\text {th }}\right)$ & $0.13(3)$ & 12 \\
\hline Canavan Disease & 19 & $0.053(23 r d)$ & $0.26(5)$ & 10 \\
\hline Down Syndrome & 48 & 0.021 (244th) & $0.083(4)$ & 24 \\
\hline Fabry Disease & 66 & 0.015 (111th) & $0.12(8)$ & 33 \\
\hline Goldblatt Syndrome & 23 & $0.043(81 \mathrm{st})$ & $0.13(3)$ & 12 \\
\hline Turner Syndrome & 26 & $0.038(21 s t)$ & $0.077(2)$ & 13 \\
\hline Uncombable Hair Syndrome & 7 & $0.14\left(1^{\text {st }}\right)$ & $0.14(1)$ & 4 \\
\hline Williams Syndrome & 180 & $0.006(121 s t)$ & $0.028(5)$ & 90 \\
\hline Yunis-Varon Syndrome & 66 & $0.015\left(7^{\text {th }}\right)$ & $0.14(9)$ & 33 \\
\hline $\begin{array}{l}\text { Zellweger-like Syndrome } \\
\text { without Peroxisomal } \\
\text { Anomalies }\end{array}$ & 25 & $0.042\left(31^{\mathrm{st}}\right)$ & $0.12(3)$ & 13 \\
\hline
\end{tabular}

11 


\section{Table 2 (on next page)}

Comparison of predictions between DDX generators.

Here we compare the most likely diagnosis of four well-known and freely available (at least for testing purposes) DDX generators with that provided by Rare Disease Discovery, when considering the joint symptoms used to perform the study summarized in columns 1 and 4 of Table 1. 
Table 2 - Comparison of predictions between DDX generators. Here we compare the most likely diagnosis of four well-known and freely available (at least for testing purposes) DDX generators with that provided by Rare Disease Discovery, when considering the joint symptoms used to perform the study summarized in columns 1 and 4 of Table 1.

\begin{tabular}{|c|c|c|c|c|c|}
\hline Disease & Diagnosis Pro & ISABEL & Phenomizer & FindZebra & $\begin{array}{c}\text { Rare Disease } \\
\text { Discovery }\end{array}$ \\
\hline Beta-Thalassemia & + & + & + & * & + \\
\hline Canavan Disease & $*$ & $*$ & + & + & + \\
\hline Down Syndrome & $*$ & $*$ & + & + & + \\
\hline Fabry Disease & + & + & + & + & + \\
\hline Goldblatt Syndrome & $*$ & $*$ & + & + & + \\
\hline Turner Syndrome & $*$ & + & + & + & + \\
\hline Uncombable Hair Syndrome & $*$ & $*$ & + & + & + \\
\hline Williams Syndrome & + & $*$ & + & + & + \\
\hline Yunis-Varon Syndrome & $*$ & $*$ & + & + & + \\
\hline $\begin{array}{l}\text { Zellweger-like Syndrome } \\
\text { without Peroxisomal } \\
\text { Anomalies }\end{array}$ & + & $*$ & + & + & + \\
\hline
\end{tabular}

+ Suggests the appropriate disease in the top 10 ranked list of predictions

* Does not suggest the appropriate disease in any position of the top 10 ranked list of predictions. 


\section{Table 3(on next page)}

Comparison of predictions between DDX generators.

Here we compare the most likely diagnosis of four well-known and freely available (at least for testing purposes) DDX generators with that provided by Rare Disease Discovery. 10 patients with different symptoms and/or diseases were randomly selected from the RAMEDIS dataset. All symptoms were used. 
Table 3 - Comparison of predictions between DDX generators. Here we compare the most likely diagnosis of four well-known and freely available (at least for testing purposes) DDX generators with that provided by Rare Disease Discovery. 10 patients with different symptoms and/or diseases were randomly selected from the RAMEDIS dataset. All symptoms were used

\begin{tabular}{|c|c|c|c|c|c|}
\hline Disease (Patient ID) & $\begin{array}{l}\text { Diagnosis } \\
\text { Pro } \\
\end{array}$ & ISABEL & Phenomizer & FindZebra & $\begin{array}{c}\text { Rare Disease } \\
\text { Discovery }\end{array}$ \\
\hline Classical homocystinuria (5) & + & ++ & ++ & ++ & ++ \\
\hline Propionic acidemia (821) & + & + & + & $*$ & ++ \\
\hline Glycogen storage disease (1086) & + & ++ & + & ++ & ++ \\
\hline Isovaleric acidemia (1050) & + & $*$ & + & ++ & ++ \\
\hline Galactosemia (970) & + & + & ++ & ++ & ++ \\
\hline Carnitine palmitoyl transferase II deficiency (1024) & $*$ & ++ & ++ & + & ++ \\
\hline Canavan disease (492) & $*$ & $*$ & $*$ & ++ & ++ \\
\hline Porphyria (866) & + & $*$ & ++ & ++ & ++ \\
\hline Mitochondrial DNA depletion syndrome (940) & $*$ & + & + & ++ & ++ \\
\hline Congenital neuronal ceroid lipofuscinosis (830) & + & + & ++ & ++ & ++ \\
\hline
\end{tabular}

+ Suggests the appropriate disease in the top 100 list of possible diseases. ++ Suggests the appropriate disease in the top 10 list of predictions.

* Does not suggest the appropriate disease in any position of the top 100 list of predictions. 